\title{
Spatial Orientation And Distribution Of Reservoir Fractures From Scattered Seismic Energy
}

Shortened title: Fracture characterization from coda waves

Mark E. Willis ${ }^{1}$, Daniel R. Burns ${ }^{1}$, Rama Rao ${ }^{1}$, Burke Minsley ${ }^{1}$, M Nafi Toksöz ${ }^{1}$, and Laura Vetri $^{2}$

1: Earth Resources Laboratory, Dept. of Earth, Atmospheric and Planetary Sciences Massachusetts Institute of Technology Cambridge, MA 02139

2: ENI E\&P, Agip, Milan Italy 


\begin{abstract}
We present the details of a new method for determining the reflection and scattering characteristics of seismic energy from subsurface fractured formations. The method is based upon observations we have made from 3D finite difference modeling of the reflected and scattered seismic energy over discrete systems of vertical fractures. Regularly spaced, discrete vertical fractures impart a ringing coda type signature to any seismic energy which is transmitted through or reflected off of them. This signature varies in amplitude and coherence as a function of several parameters including: 1) the difference in angle between the orientation of the fractures and the acquisition direction, 2) the fracture spacing, 3) the wavelength of the illuminating seismic energy, and 4) the compliance, or stiffness, of the fractures. This coda energy is the most coherent when the acquisition direction is parallel to the strike of the fractures. It has the largest amplitude when the seismic wavelengths are tuned to the fracture spacing, and when the fractures have low stiffness. Our method uses surface seismic reflection traces to derive a transfer function which quantifies the change in an apparent source wavelet before and after propagating through a fractured interval. The transfer function for an interval with no or low amounts of scattering will be more spike-like and temporally compact. The transfer function for an interval with high scattering will ring and be less temporally compact. When a 3D survey is acquired with a full range of azimuths, the variation in the derived transfer functions allows us to identify subsurface areas with high fracturing and determine the strike of those fractures. We calibrated the method with model data and then applied it to the Emilio field with a fractured reservoir giving results which agree with known field measurements and previously published fracture orientations derived from PS anisotropy.
\end{abstract}

Keywords: fracture, scattering, coda, scattering index

\title{
INTRODUCTION
}

Evidence continues to confirm that much of the earth's crust, especially below a critical depth of 500 to 1000m, contains a predominance of nearly vertical fractured rocks (Crampin and Chastin, 2000) typically aligned subparallel to the regional direction of maximum compression or about 45 degrees to the axis of principal stress (Crampin et al, 1980). These natural fracture systems in an oil and gas reservoir frequently dominate the fluid 
drainage pattern, turning hydrocarbon saturated rocks with even low matrix permeability into significant commercial assets. In many low permeability oil fields, hydraulic fracturing is undertaken to enhance the natural system of fractures and increase production rates (e.g. Block et al, 1994; Fehler et al, 1998; Phillips et al, 1998; House et al, 2004). An understanding of these fracture systems is crucial for field development planning in order to more completely drain the reservoir from the fewest number of wells.

Seismic waves traveling through a rock formation containing aligned fractures are affected by the fractures' mechanical parameters, such as compliance and saturating fluid, and on their geometric properties. If the fracture dimensions and spacing are small relative to the seismic wavelength, then the fractures cause the reservoir rock to behave like an equivalent anisotropic medium with a symmetry axis normal to the strike of the 'open' fractures. Resulting seismic reflections from the top and bottom of a fractured reservoir will display amplitude variations with offset and azimuth (AVOA). In recent years much progress has been made analyzing AVOA effects (e.g., Lynn et al., 1996; Sayers and Rickett, 1997; Perez et al, 1999; Shen and Toksöz; 2000; Jenner, 2002; Shen et al, 2002; Hall and Kendall, 2003; Lynn and Cox, 2003; Minsley et al, 2004).

If, however, the fracture dimensions and spacing are close in size to the seismic wavelength, then the fractures will scatter the P- and converted S-wave energy causing a complex, reverberating, seismic signature or coda. This seismic signature will vary as a function of the orientation of the seismic acquisition relative to the fracture orientation. Work by several authors (e.g. Ata and Michelena, 1995; Schultz and Toksöz ,1996; Daley et al, 2002; Nakagawa et al, 2002; Wu et al, 2002; Nakagawa et al, 2003; Willis et al, 2004a) using ultrasonic scale modeling and numeric simulation have demonstrated complicated, azimuthally varying scattering patterns by simulating systems of subsurface, aligned fractures. The scattered seismic energy not only provides information about the fracture orientation, but can also be analyzed to provide information about the fracture spacing (Willis et al, 2004a) and fracture density (Pearce, 2003).

In this paper we describe our recent work (Willis et al, 2003; Willis et al, 2004a; Willis et al, 2004b; Willis et al, 2004c; and Burns et al, 2004) to extract fracture distribution and orientation from scattered coda waves where the fracture systems are of a size comparable to the wavelength of the seismic source. We first describe our modeling 
results of vertically fractured reservoirs, our methods to extract the fracture properties from surface reflection seismic acquisition data, and finally the results on field data.

\section{MODELING}

We model a simple reservoir using the 3-D anisotropic, elastic, finite-difference code developed by Lawrence Berkeley National Laboratory (Nihei et al., 2002). The code implements the algorithm described by Levander (1988) which uses a staggered grid with an explicit, fourth order operator in space and a second order operator in time. The model geometry (Figure 1) and parameters (Table 1) we used consists of five horizontal layers. All the layers except the middle, third layer, are homogeneous and isotropic elastic media. The background medium for the third layer is isotropic and homogeneous. We want to simulate a periodic series of parallel, vertical fractures inserted into this layer. So we use the Coates and Shoenberg (1995) method to represent the fractures by grid cells containing equivalent anisotropic medium. Vlastos et al (2003) have recently use this same approach in a 2-D pseudospectral approach for modeling scattering from fractures.

Following Daley et al (2002), we use normal and tangential fracture stiffness values of $8 \times 10^{8} \mathrm{~Pa} / \mathrm{m}$ representing long, compliant, gas-filled fractures. The grid cells containing the fractures are chosen to be vertical planes, a single grid cell thick (5m), as tall as the layer thickness $(200 \mathrm{~m})$ which run the entire width of the model (i.e. parallel to $\mathrm{x}=0$ ). We generated a series of models with the following regular fracture spacings: no fractures, $10 \mathrm{~m}, 25 \mathrm{~m}, 35 \mathrm{~m}, 50 \mathrm{~m}$, and $100 \mathrm{~m}$. We also generated another model to insure that our results would not be restricted to perfectly regular fracture spacings. The model has a Gaussian distribution of vertical, parallel fractures with a mean spacing of 35m and a standard deviation of $10 \mathrm{~m}$.

On the left side of Figure 2 are the shot records for the $50 \mathrm{~m}$ fracture spacing case acquired in directions normal (top) and parallel (bottom) to the fractures. The P wave reflections off the top of layers two and three arrive at zero offset times of about 170 and $290 \mathrm{~ms}$, respectively. The arrival at $220 \mathrm{~ms}$ is the converted S wave reflected off the top of layer two. Below these three distinct arrivals, are a series of events corresponding to the scattering from the fractures. 
To the right of each shot record in Figure 2, is its semblance-based, stacking-velocity analysis. Since the model interval P velocities are all above $2900 \mathrm{~m} / \mathrm{s}$, it is clear that the shot record normal to the fracture direction (top) has very little coherent and stackable P energy below the top of the reservoir level (290 ms),. However, for the parallel case (bottom), there are many coherent events below the top reservoir reflection. In the direction parallel to the fractures, the seismic energy seems to be guided by the aligned fractures and the resulting scattered energy is more coherent and similar to the direct $\mathrm{P}$ wave reflection. This same pattern of azimuthal variation in the modeled scattered wavefields is observed for all the model results regardless of the fracture spacing. For the dual fracture model, this same pattern is seen for the primary facture set but the effect of the secondary fracture set is not very large.

For shot records acquired parallel to the fractures, the ringing scattered events are seen to be the most coherent on the near through mid offset ranges. Figure 3a shows ten azimuth stacks. They were created by applying normal move out and stack to different azimuth ranges of the model traces starting in the direction normal to the fractures, then rotating by 10 degree increments, until finally parallel to the fractures. The trace labeled "normal” corresponds to the stack of the traces in the top left plot of Fig. 2. The trace labeled "parallel" corresponds to the stack of traces in the bottom left plot of Fig 2. These stacks do not include the far offset traces. For comparison, the bottom trace labeled "control" is the stacked trace from the model without fractures. For shot records acquired normal to the fracture direction, the observed scattered wave field is greatly disruptive with significant back scattered, diffractionlike events. These traces do not stack together well with any NMO velocity. However, the traces acquired parallel to the fractures stack in considerably better. Based upon these observations, the strike of the fracturing may be determined by identifying the acquisition direction with shot records containing coherent, ringing energy which are enhanced the most when stacked. Figure 4a shows the azimuth stacks for the other models studied. This same trend is present for all models except the $10 \mathrm{~m}$ fracture spacing case. For this model the fracture spacing is so small that the third layer behaves more like an equivalent anisotropic medium than one with large scale fracturing. 


\section{EXTRACTING SOURCE WAVELET AND COMPUTING TRANSFER FUNCTION}

In the synthetic traces we generated, it is possible to directly observe the scattered waves and their azimuthally varying trends. This is due to the fact that there are only a few, isolated reflectors in the model. However, in field data we expect to have a more difficult time clearly observing these scattered wave trains due to the nearly continuously changing nature of subsurface reflectivity and the potentially lower amplitudes of the scattered energy. Figure 5 is a schematic showing that reflections off beds shallower than a fractured zone will not be affected by it. However, reflections from below it will acquire a ringing coda caused by reverberations in the fractured zone. In addition, if the overburden is factured, those scattered waves will contaminate, or overprint, the scattered energy from the zone of interest in the reservoir. Thus the problem we face is detecting the change in the reflection character of an apparent source wavelet as we move down each trace. Specifically, we want to detect the change in the temporal "compactness" of the apparent source wavelet as it passes through each formation of interest.

Traditional methods of source wavelet extraction are based upon the notion of the stationarity of the seismic wavelet, which means that the source wavelet doesn't change with time down the trace (Yilmaz, 1987). For our purposes, we assume stationarity only within each time window used to estimate the source wavelet. However, due to the mode conversions and reverberation in the fractured interval, the apparent source wavelet does change with time down the trace. So we extract two apparent source wavelets from the reflection time series - one from above the proposed fractured zone (the "input" wavelet) and one below it (the "output” wavelet). These wavelets are represented by their autocorrelations obtained from windowed portions of the reflection time series above and below the fractured zone of interest. We make the standard assumption that the reflectivity series is white. Hence, the autocorrelation of the windowed time series yields the autocorrelation of the source wavelet in that window.

We then compute the time domain transfer function (sometimes called the impulse response) between the autocorrelations of the two extracted wavelets. The transfer function is computed by deconvolving the autocorrelation of the input wavelet from the autocorrelation of the output wavelet using the Weiner-Levinson algorithm (Robinson and Treitel, 1980). Alternatively, another deconvolution method like spectral division could be used. The transfer function characterizes the effect of scattering in the interval of interest between the two windowed 
portions of the trace. Since both the input and output autocorrelations are zero phase, the resulting transfer function will also be zero phase and symmetric. A simple spike or pulse shaped transfer function indicates no scattering, while a long ringing transfer function reveals that scattering has occurred within the proposed interval between the analysis windows. This measurement will be insensitive to contamination from an acquisition footprint or from scattering in the overburden. This is due to the fact that these effects would appear on both the input and output extracted wavelets and thus will be excluded from the transfer function.

The transfer function can be used to characterize scattering on both pre- and post-stack data. On pre-stack data it can detect the presence of scattering on a single trace. However, it can also be used to determine the orientation of fracturing by comparing the change in the transfer functions from stacked traces with different acquisition orientations. The transfer functions from traces stacked in the direction parallel to fracturing will exhibit more ringing than those in the direction normal to fractures. To show this on the 50m fracture spacing model data, we choose the input and output time windows on each of the traces in Figure 3b, as delineated by the labeled bars beneath the traces. We form the autocorrelation of each window and then compute the transfer function between each corresponding pair of autocorrelations. Figure 3b shows the derived transfer functions. Notice that the transfer function for the control case, the model without fractures, is very impulsive and is similar to a band limited spike. The transfer function for the stacked normal trace strongly resembles the control case. The transfer functions show very little change in shape until they are within 10 degrees of the fracture strike direction, indicating a sharp angular resolution. The transfer function for the parallel trace rings for about $100 \mathrm{~ms}$ in each direction and is very different from the other functions.

We have applied this analysis to all of the other models and the results are shown in Figure $4 \mathrm{~b}$. In all cases except the $10 \mathrm{~m}$ fracture case, the transfer functions ring most prominently in the direction parallel to the fracturing. (As discussed earlier, the $10 \mathrm{~m}$ fracture case behaves more like an equivalent anisotropic medium than one that shows discrete fracturing.) From these examples it is clear that the marked ringing behavior of the transfer function for the stacks in the direction parallel to the fractures is a characteristic phenomenon and not an artifact of a random perfect resonance in a particular model. Therefore the transfer functions can be used to estimate fracture orientation. 


\section{METHODOLOGY FOR SCATTERING INDEX}

We have shown that stacks made from traces acquired parallel to a prominent fracture system retain the ringing scattered coda energy on the traces. Stacks made in other acquisition directions tend to diminish the scattered coda energy. This same trend is evident on the corresponding transfer functions. By design, a transfer function is symmetric about zero lag so we only need to examine its positive time lags. Looking more closely at the transfer functions for orientations parallel to fractures, we clearly see the ringing coda creates energy in the transfer function at times away from the zero lag. However, in the normal direction the transfer functions are comparatively compact about the zero lag.

In order to quantify the amount of ringing or non-compactness in a transfer function, we define a scattering index, SI, with a form given by:

$$
S I=\sum_{i=0}^{m}\left|t_{i}\right| i^{n}
$$

where $\mathrm{i}$ is the time lag, $\mathrm{t}_{\mathrm{i}}$ is the transfer function (time domain) amplitude at lag $\mathrm{i}, \mathrm{n}$ is an exponent, typically equal to unity, and $\mathrm{m}$ is a lag at which there is no more significant energy in the transfer function. (It is also possible to normalize the scattering index based upon its energy and interval time sample or other such criteria.) This expression weights the large lag times more heavily than the near zero lag times in the transfer function. The more the transfer function rings, the larger the value of the scattering index. If the transfer function is a simple spike, i.e. representing no scattering, then the scattering index attains a value of zero.

Figure 6 shows the scattering index values for the models with 25, 35, 50 and 100m fracture spacings. The extent of the model doesn't afford a complete 360 degree acquisition direction analysis so we've replicated appropriately the first quadrant analysis for the other three quadrants. These results show that there is a clear maximum of the scattering index in the parallel direction. It is also clear that in the non-parallel directions the scattering index is not zero but fluctuates about a smaller, but somewhat consistent value. The scattering index 
formulation allows us to extract the amount of ringing in the transfer functions as a single digit making it easier to analyze, display and therefore detect the strike of the fracturing.

\section{Results on field data}

In early 2000, a 3D/4C seismic survey was collected over the Emilio Field, located in the central part of the Adriatic Sea, near the eastern coast of Italy. The reservoir unit is a fractured carbonate which from borehole studies suggests the presence of two orthogonal fracture sets oriented ENE and NNW (Angerer et al, 2002). Recent studies have investigated this 3D seismic data using PP and PS wave anisotropy to identify fracture characteristics of the reservoir level (Vetri et al, 2003; Gaiser et al, 2002). Figure 7 (taken from Figure 10 of Vetri et al, 2003) shows the interval velocity log, the interpreted seismic section and structural model of a profile through the field. The most prominent reflector in the section is the Gessosso-Soliferera, highlighted in green, which is a high velocity chalk formation. The reservoir interval is shown between the cyan and purple lines on the seismic section.

We stacked the near to mid range $(<3500 \mathrm{~m})$ offsets of the preprocessed PP data (Vetri et al, 2003) in eighteen different azimuth orientations from East to West using 20 degree wide overlapping ranges, in 10 degree steps. (Note that these angle ranges included the corresponding ranges 180 degrees away.) This process created eighteen 3D stacked volumes. The transfer functions and scattering indices for the formation zone were computed for each of these stacked volumes. The scattering indices were sorted and directions for those with the highest angular contrast in values (differences $>5$ ), are plotted as quivers in Figure 8, giving a map view of the location and direction of possible fractures determined by this method.

The locations of the fracture measurements are taken from stacked data, and as such, their locations are in the un-migrated positions. To adjust for this potential mis-positioning, we performed a map migration of all the scattering indices with angular contrasts greater than 4 . These results are shown as blue quivers in Figure 9. Here we have used the coordinate system of inlines and cross lines (rather than Northings and Eastings in Figure 8) to be able to plot the seismically derived fault system (in black) and well information on the same diagram. We observe 
that the clusters of the blue quivers tend to congregate around the fault zones. In addition, we see the quivers tend to align either parallel or perpendicular to the faulting.

The left side of Figure 10 shows a rotated version of Figure 9, to align with the Northings and Eastings coordinate system. The right side of Figure 10 is a plot of the scattering directions for all the CDP locations in the survey, without omitting low angular contrast values. The scattering index directions have been color coded using the color legend on the top right of the figure. Small angular contrasts in the scattering indices are denoted by tinting the color of the corresponding CPP cells toward the center of the color legend wheel which is white. Larger angular contrasts are tinted toward brighter colors at the edge of the legend color wheel indicating greater confidence. Green, Red, Gray and Blue colors indicate fracture strikes of East, Northeast, North and Northwest, respectively.

We next compare our fracture directions with those derived by shear wave anisotropy. The left panel of Figure 11 (modified from Figure 10 of Vetri et al, 2003) shows the fracture strike direction derived from the fast direction of the PS waves. We have added three black arrows to help interpret their color scale. In the right panel of Figure 11 we have taken our fracture directions (from Figure 10) and performed modal smoothing of the directions using a 75x150m box centered about each CDP. We also plotted three arrows indicating three fracture direction trends. The mostly red, large area in the lower part of the right panel is the most obvious feature. This area indicates a fracture direction of NE which is identical to the direction indicated by the shear wave data. Close comparison of the figures shows that the directions agree over much of the survey.

The final result in shown in Figure 12 comparing well derived fracture orientations with those derived by the scattering index analysis. The top row shows the well information where in general fracture strike directions are parallel to the SH maximum direction. The bottom row shows close-ups from Figure 9 around these three wells. It is clear that there is good agreement of the scattering index derived orientations at all wells. 


\section{CONCLUSIONS}

Large-scale zones of fracturing control fluid flow in certain reservoirs, and such zones can scatter seismic energy depending on the fracture density and compliance, as well as the spacing relative to the seismic source wavelength. This scattered wave energy contains information about the fracture properties. Using numerical modeling data we developed a method of analyzing scattered wave energy from fractured reservoirs. A deconvolutional process that measures the 'ringiness' of the transfer function can be used to estimate fracture orientation from azimuthally stacked data. The application of this method on field data provide fracture orientation estimates which agree very closely with previous borehole and PS anisotropy studies in the Emilio field. The value of this methodology is that is a very simple, easy to parameterize algorithm that should not be prone to overburden effects. Its application on both model and field data show that it is both robust and accurate. 


\section{REFERENCES}

Angerer, E, Horne, S, Gaiser, J., Walters, R., Bagala, S., 2002, Characterization of dipping fractures using Ps modeconverted data, SEG Expanded Abstracts.

Ata, E., and Michelena, R. J., 1995, Mapping distribution of fractures in a reservoir with P-S converted waves, The Leading Edge, v. 12, 664-676.

Block, L, Cheng, C.H., Fehler, M., and Phillips, W.S., 1994, Seismic imaging using microearthquakes induced by hydraulic fracturing, Geophysics 59, p. 102-112.

Burns, D, Willis, M., Minsley, B., and Toksoz, M.N, 2004, Characterizing subsurface fractures from reflected and scattered seismic energy, Jap. SEG Ann. Meeting.

Coates, R. T., and Schoenberg, M., 1995, Finite-difference modeling of faults and fractures, Geophysics, 60, n5, 1995

Crampin, S. and Chastin, S., 2000, Shear-wave splitting in a critical crust: II - compliant, calculable, fluid-rock interactions, in Anisotropy 2000: Fractures, converted waves and case studies, Soc. Expl. Geophys. Tulsa.

Crampin, S., McGonigle, R. and Bamford, D, 1980, Estimating crack parameters from observations of P-wave velocity anisotropy, Geophysics, 45 (3), p 345-360.

Daley, T. M., Nihei, K, Myer, E., Queen, J, Fortuna, M, Murphy, J and Coates, R., 2002, Numerical modeling of scattering from discrete fracture zones in a San Juan Basin gas reservoir, $72^{\text {nd }}$ Ann. Int. Mtg. Soc. Expl. Geophys. Expanded Abstracts.

Fehler, M., House, L, Phillips, W.S., and Potter, R., 1998, A method to allow temporal variation in travel-time tomography using microearthquakes induced during hydraulic fracturing, Tectonophysics 289, p. 189-201.

Gaiser, J., Loinger, E., Lynn, H, and Vetri, L, 2002, Birefringence analysis at the Emilio field for fracture characterization, First Break v 20, 505-514.

Hall, S. and Kendall, J-M, 2003, Fracture characterization at Valhall: application of P-wave amplitude variation with offset and azimuth (AVOA) analysis to a 3D ocean-bottom data set, Geophysics 68, 1150.

House, N, Fuller, B, Shemeta, J, 2004, Integration of surface seismic, 3D VSP, and microseismic hydraulic fracture 
mapping to improve gas production in a tight complex reservoir, $74^{\text {th }}$ Ann. Mtg Soc. Of Explor. Geophys. Expanded Abstr. Pp 414-416.

Jenner, E., 2002, Azimuthal AVO: methodology and data examples, The Leading Edge 21, 782.

Levander, A. R., 1988, Fourth-order finite-difference P-SV seismograms. Geophysics, 53, 1425-1436.

Lynn, H and Cox, D, 2003, P-wave AVOA interpretation needs the input of additional information, SEG Expanded Abstracts 22, 124.

Lynn, H., Simon, K. M., and Bates, C. R., 1996, Correlation between P-wave AVOA and S-wave traveltime anisotropy in a naturally fractured gas reservoir, The Leading Edge, v 15, 931-935

Minsley, B, Willis, M, Krasovec, M, Burns, D, and Toksöz, M.N. 2004, Investigation of a fractured reservoir using P-wave AVOA analysis: a case study of the Emilio Field with support from synthetic examples, SEG Expand. Abstr. 23, 248.

Nakagawa, S, Nihei, K, and Myer, L., 2002, Numerical simulation of 3D elastic wave scattering off a layer containing parallel periodic fractures, SEG Expand. Abstr.

Nakagawa, S, Nihei, K, and Myer, L., 2003, Three-dimensional elastic wave scattering by a layer containing vertical periodic fractures, JASA, 13 (6).

Nihei, K., Nakagawa, S., Myer, L. and Majer, E., 2002, Finite difference modeling of seismic wave interactions with discrete, finite length fractures, $72^{\text {nd }}$ SEG Expanded Abstracts.

Pearce, F, 2003, Seismic scattering attributes to estimate reservoir fracture density: a numerical modeling study, M.S. Thesis, MIT.

Perez, M., Gibson, R. and Toksoz, M.N, 1999, Detection of fracture orientation using azimuthal variation of P-wave AVO response, Geophysics 64, 1253-1265.

Phillips, W., Fairbanks, T., Rutledge, J. and Anderson, D, 1998, Induced microearthquake patterns and oilproducing fracture systems in the Austin chalk, Tectonophys 298, p 153-169.

Robinson, E, and Treitel, S., 1980, Geophysical signal analysis, Prentice Hall Inc. 
Sayers, C.M. and Rickett, J., 1997. Azimuthal variation in AVO response for fractured gas sands, Geophysical Prospecting, 45, 165

Schultz, C. and Toksöz, M.N., 1996, Experimental study of enhanced backscattering from a highly irregular, acoustic-elastic interface, J. Acoust. Soc. Am., 99, 880-892.

Shen, F and Toksöz, M.N., 2000, Scattering characteristics in heterogeneously fractured reservoirs from waveform estimations, Geophys. J. Int. 140, 251-266.

Shen, F., Sierra, J., Burns, D. R., and Toksoz, M.N., 2002, Azimuthal offset-dependent attributes applied to fracture detection in a carbonate reservoir, Geophysics, v.67, no.2, p.355-364.

Vetri, L., Loinger, E. Gaiser, J. Grandi, A., Lynn, H, 2003, 3D/4C Emilio: Azimuth processing and anisotropy analysis in a fractured carbonate reservoir, The Leading Edge, 675-679.

Vlastos, S., Liu, E., Main, I.G. and Li, X-Y, 2003, Numerical simulation of wave propagation in media with discrete distributions of fractures: effects of fracture sizes and spatial distributions, Geophys. J. Int., 152, pp 649-668.

Willis, M.E., Burns, D.R, Rao, R. and Minsley, B, 2003, Characterization of scattering waves from fractures by estimating the transfer function between reflected events above and below each interval, Annual Sponsors' Meeting of MIT/ERL.

Willis, M.E., Pearce, F, Burns, D.R, Byun, J. and Minsley, B, 2004a, Reservoir fracture orientation and density from reflected and scattered seismic energy, EAGE meeting Paris.

Willis, M.E., Burns, D, Rao, R, Minsley, B, and Zhang, Y, 2004b, Characterizing seismic scattering from discrete fracture systems, SEG Research Workshop on Fractured Reservoirs, Vancouver.

Willis, M.E., Rao, R., Burns, D., Byun, J, and Vetri, L, 2004c, Spatial orientation and distribution of reservoir fractures from scattered seismic energy, $74^{\text {th }}$ SEG Expanded Abstr., Denver.

Wu, C, Harris, J. and Nihei, K, 2002, 2-D finite-difference seismic modeling of an open fluid-filled fracture: comparison of thin-layer and linear-slip models, $72^{\text {nd }}$ Ann. Mtg SEG Expand. Abstr.

\section{ACKNOWLEDGEMENTS}


Joongmoo Byung and Fred Pearce ran the finite difference models used in this paper. Yang Zhang created the point diffractor modeling results. Our thanks to Lawrence Berkley Lab for use of their 3D finite difference modeling code. We would like to recognize and thank ENI S.p.A. AGIP, the Department of Energy Grant number DE-FC2602NT15346, and the Earth Resources Laboratory Founding Member Consortium for funding and supporting this work. 
Table 1. Parameters for model

\begin{tabular}{|c|c|c|c|c|}
\hline Layer & Thickness (m) & Vp (m/s) & Vs (m/s) & Density (g/cc) \\
\hline 1 & 200 & 3000 & 1765 & 2.2 \\
\hline 2 & 200 & 3500 & 2060 & 2.25 \\
\hline 3 & 200 & 4000 & 2353 & 2.3 \\
\hline 4 & 200 & 3500 & 2060 & 2.25 \\
\hline 5 & 200 & 4000 & 2353 & 2.3 \\
\hline
\end{tabular}




\section{FIGURE CAPTIONS}

Figure 1. Diagram showing the geometry of the 3D finite difference model. The dimensions of the model, not including the absorbing boundaries, are 1875m in $\mathrm{x}$ and $\mathrm{y}$ and $1350 \mathrm{~m}$ in depth, $\mathrm{z}$. The layer velocities and densities are shown in Table 1. The source is located in the left front corner (red symbol) and the receivers are spread out in a rectangular area $1000 \mathrm{~m}$ in the $\mathrm{x}$ direction and $1000 \mathrm{~m}$ in the $\mathrm{y}$ direction. The receiver spacing is $5 \mathrm{~m}$ in each direction.

Figure 2: Left two plots show the seismic shot records for the model with 50m fracture spacing. The top left plot show the shot record normal to fractures, left bottom plot shows the shot record parallel to the fractures. The right two plots show the velocity spectra for the corresponding shot records on the left.

Figure 3: a) Azimuthal stacks of traces from the 50m fracture spacing model. The traces represent azimuth stacks starting in the direction parallel to fracturing (top), and then increasing in 10 degree increments until normal to the fractures. The bottom trace shows the stack for the model without a fractured layer. b) Corresponding transfer functions.

Figure 4: a) Azimuthal stacks of traces from models with various fracture spacing . b) Corresponding transfer functions.

Figure 5. Schematic showing that the reflected energy from layers above a fractured zone will not be altered by the

fractures. However, the energy reflected off layers below the fractured zone will acquire a ringing coda caused by fractures.

Figure 6: Polar plot of the azimuthal variation of scattering indices derived from the transfer functions of the 25, 35, 50 and $100 \mathrm{~m}$ fracture spacing models. The scattering index is largest in the direction parallel to the fracture 
orientation. The largest scattering index is for the $35 \mathrm{~m}$ fracture spacing, while the smallest shown is for the $25 \mathrm{~m}$ spacing.

Figure 7. Profile through the Emilio PP data showing interval velocity log (left), interpreted seismic section (middle) and structural model (right) from Vetri et al, 2003.

Figure 8. Fracture orientations for the Emilio field from scattering index values showing an angular contrast in values larger than 5 .

Figure 9. Map-migrated scattering index fracture directions (in blue) for the Emilio field (having angular contrast values $>4$ ). The black lines indicate faults derived from seismic data. The well locations are indicated by the round colored circles.

Figure 10. A comparison of the map-migrated scattering index fracture orientations (left panel copied and rotated from Figure 9) having high angular contrast values with scattering index fracture directions for all CDPs (right panel). The color legend at the top right indicates the fracture direction in hue and increasing angular contrast with intensity.

Figure 11. The left panel shows the fracture strike derived from PS anisotropy (Vetri et al, 2003) with its corresponding color-coded direction legend at the bottom left. The right panel shows the smoothed fracture orientations derived from the scattering index analysis in Figure 10 with its corresponding color-coded legend at the top right. The black arrows indicate three fracture orientation trends. 
Figure 12. The top set of diagrams show the well derived fracture information - SHmax is generally the direction of fracture strike. The bottom three diagrams are close-ups of Figure 9 about the corresponding well locations showing the agreement with the well fracture directions. 


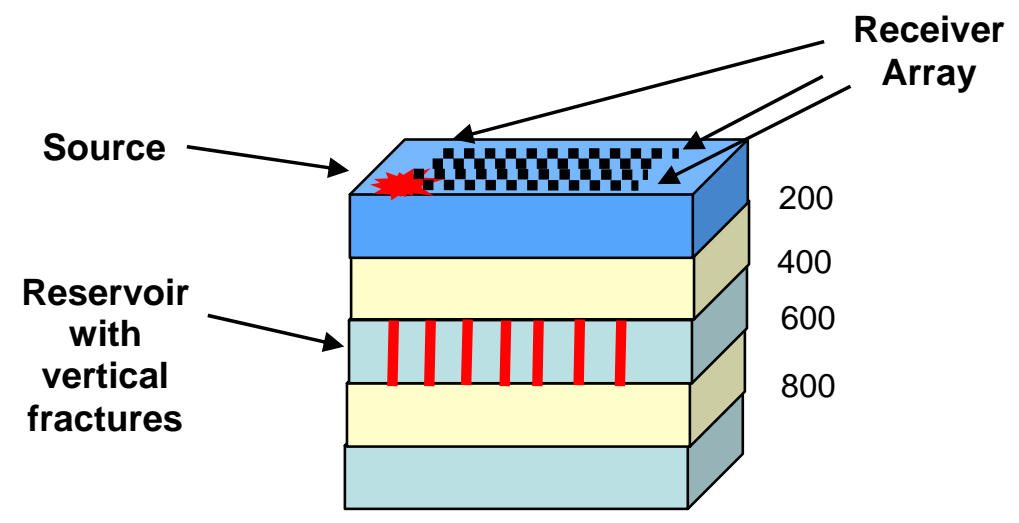

Figure 1. 

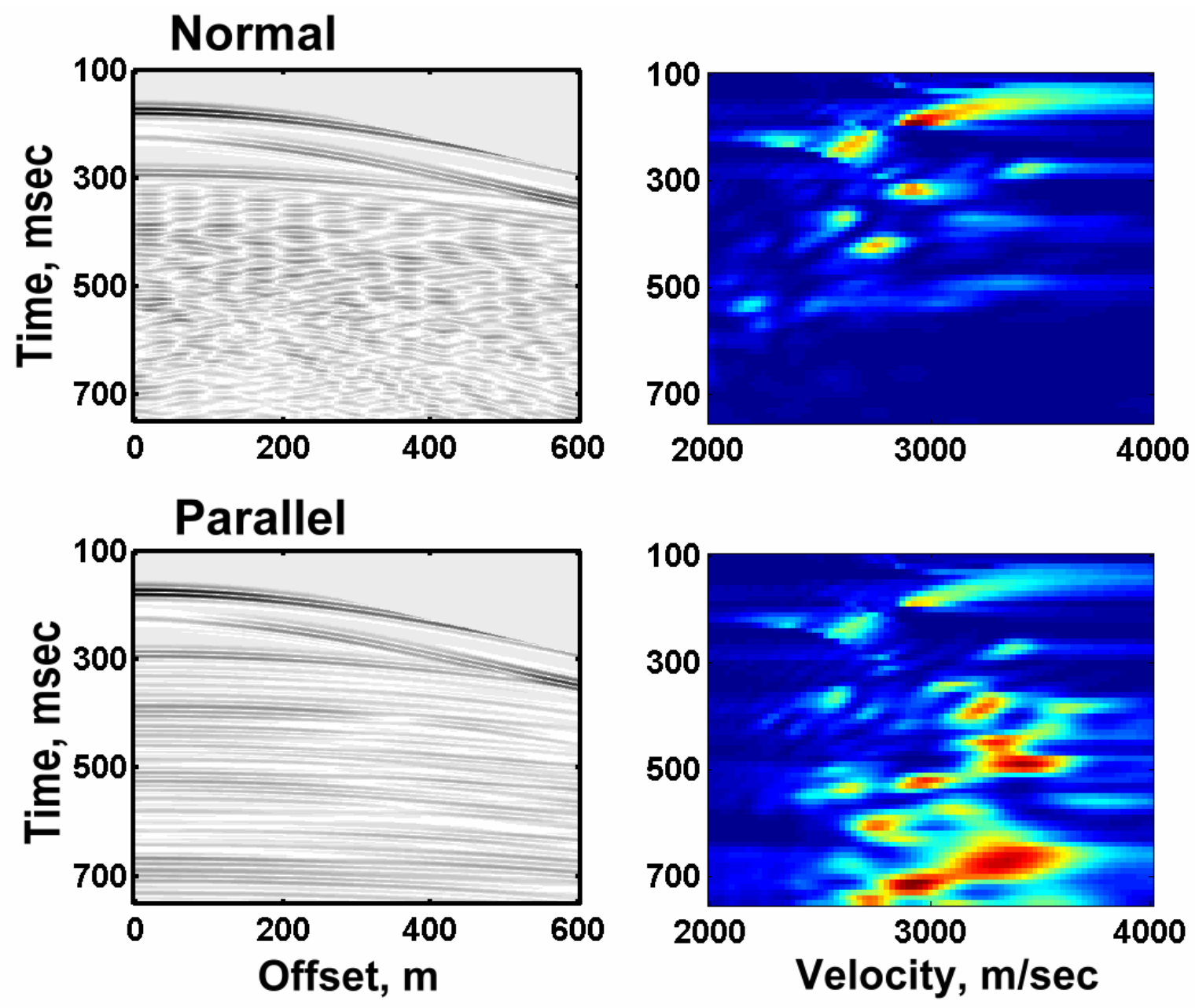

Figure 2. 
Azimuthal Stacks - 50m Fracture Spacing

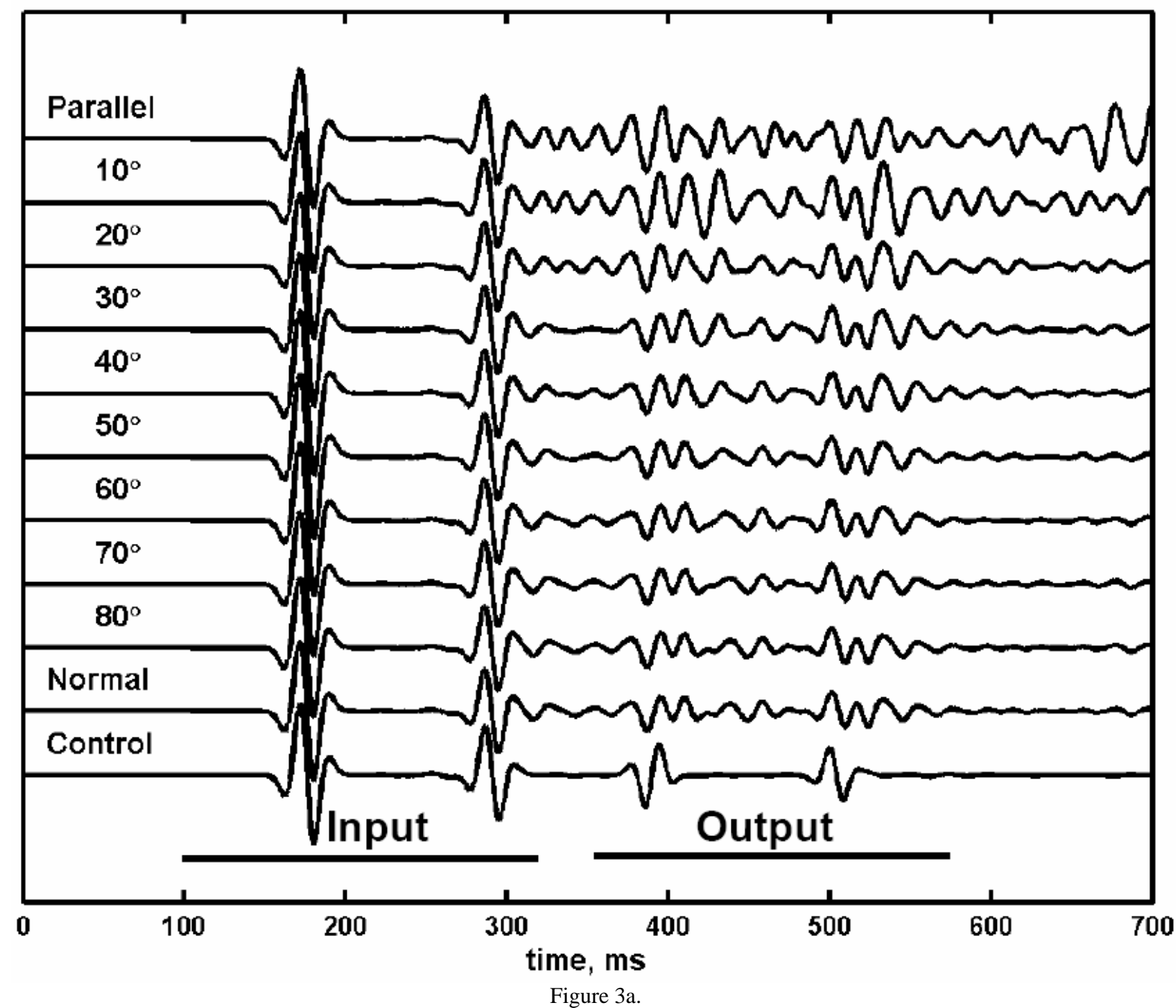




\section{Azimuthal Stack Transfer Functions - 50m Fracture Spacing}

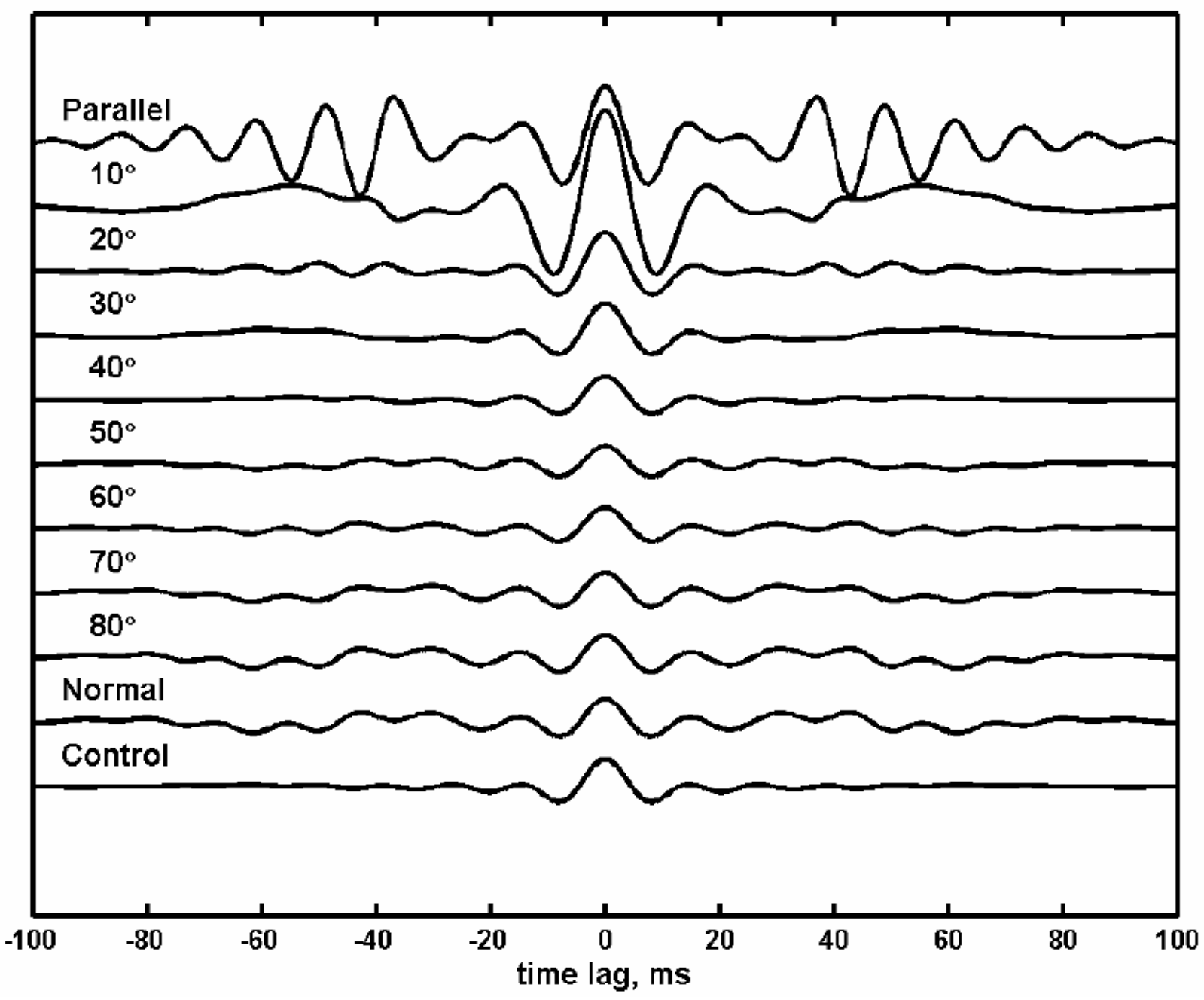

Figure 3b. 
$100 \mathrm{~m}$

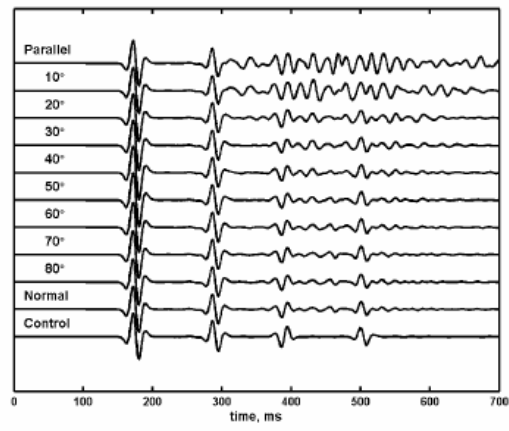

\section{$35 \mathrm{~m}$}

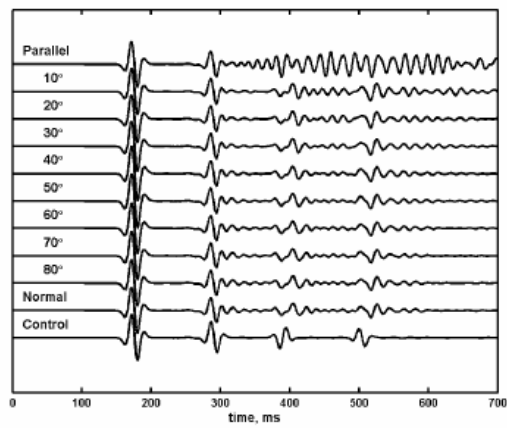

$25 m$

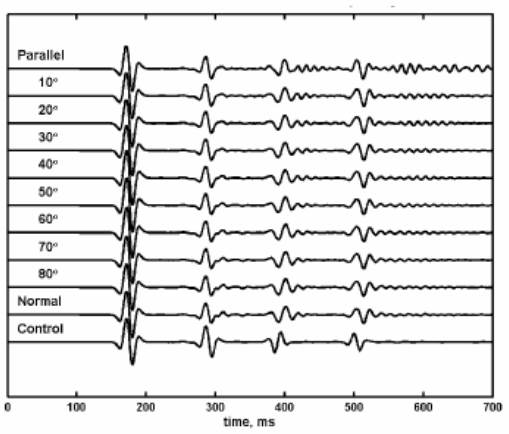

35m Gaussian

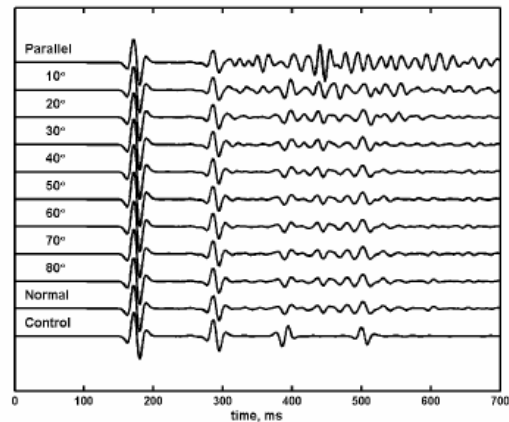

Figure 4a.
$10 \mathrm{~m}$

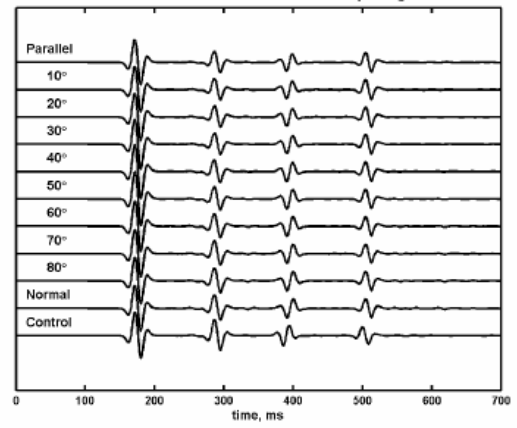



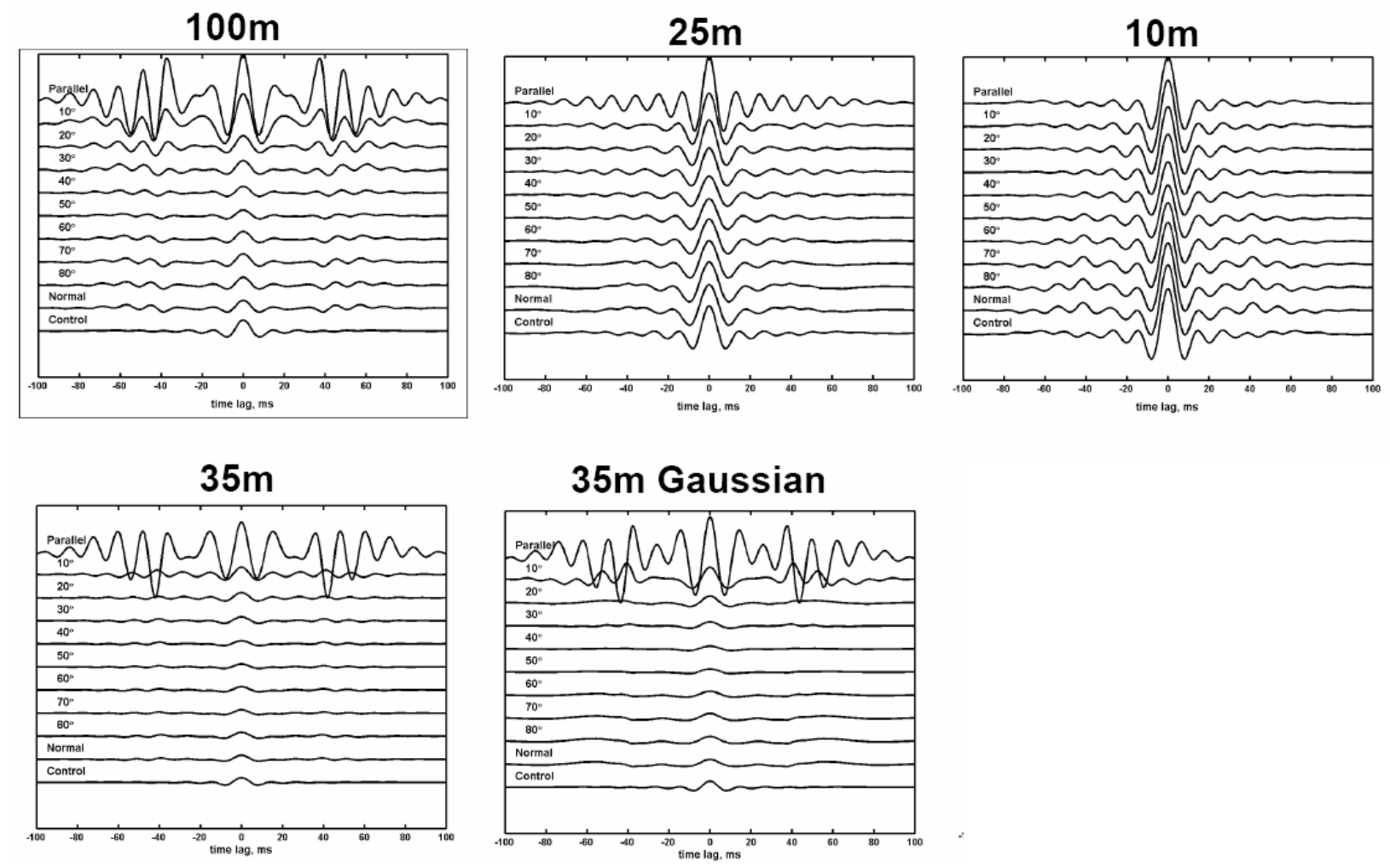

Figure 4b. 


\section{Down Going Seismic Energy}

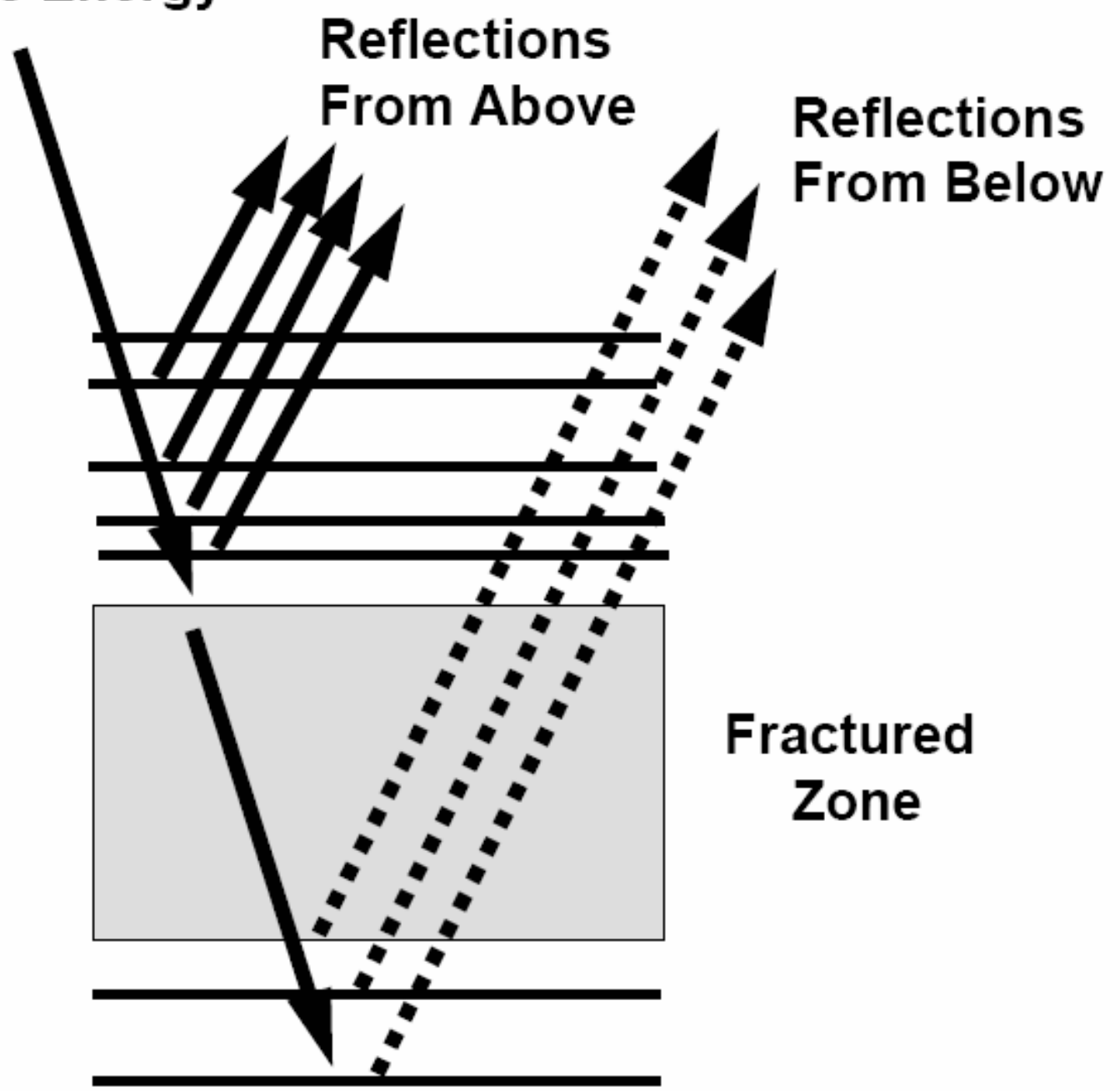

Figure 5. 
Azimuthal Scattering Indices for Four Fracture Spacings

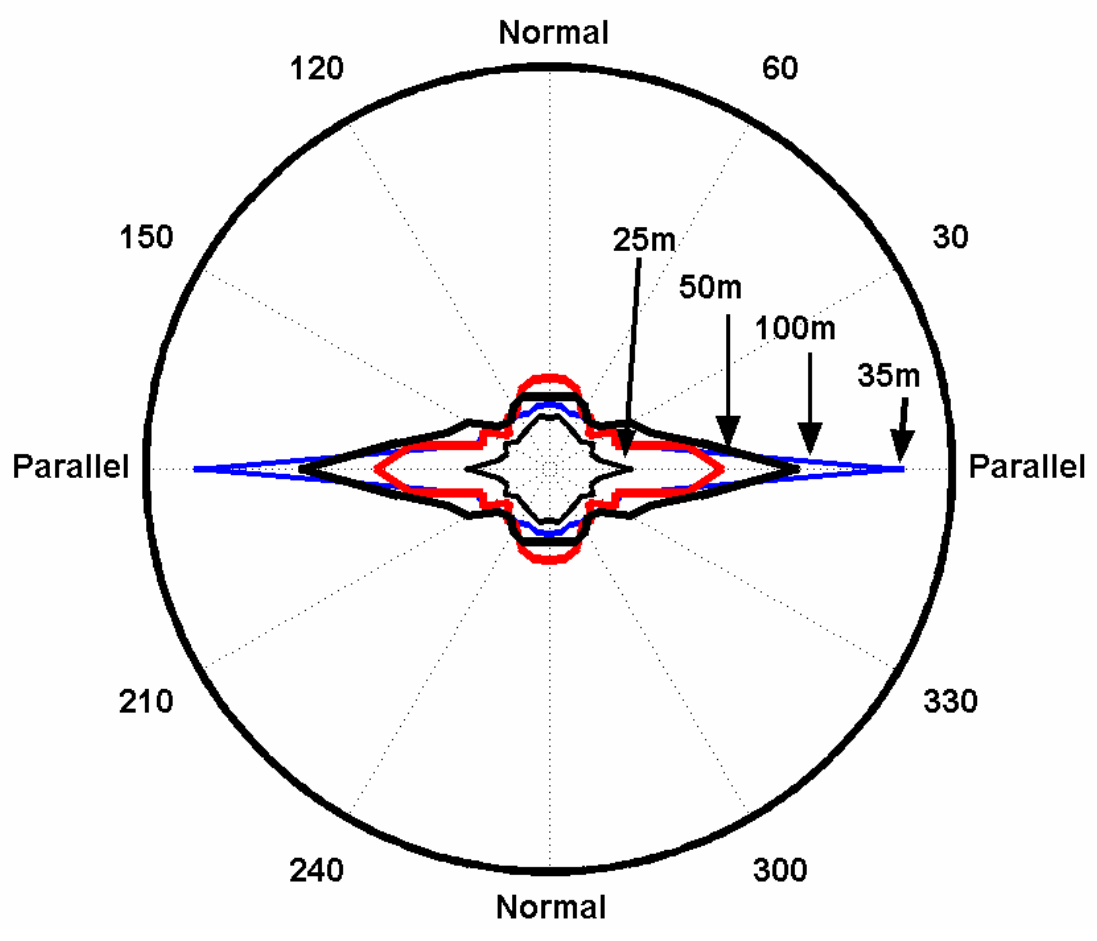

Figure 6. 


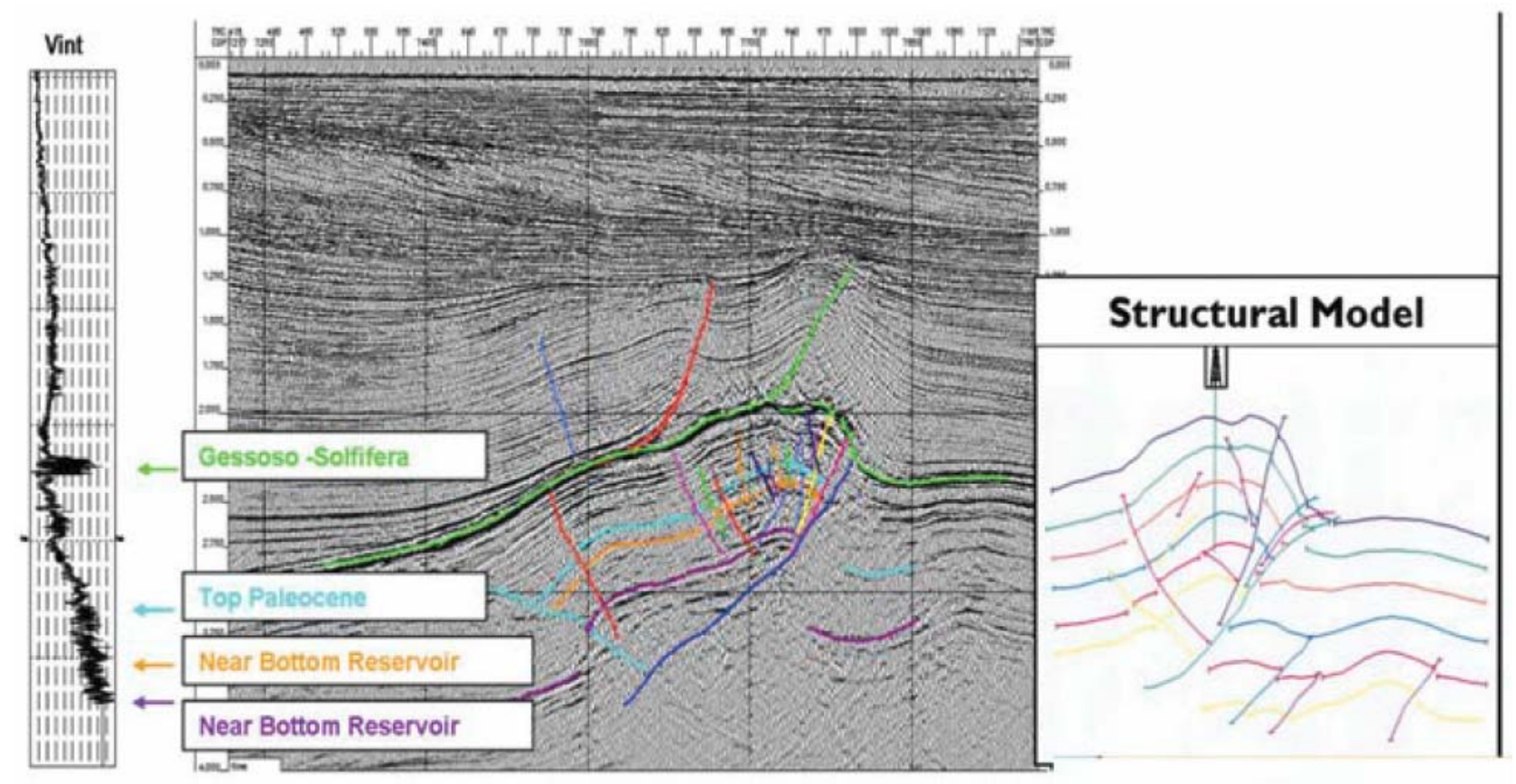

Figure 7. 


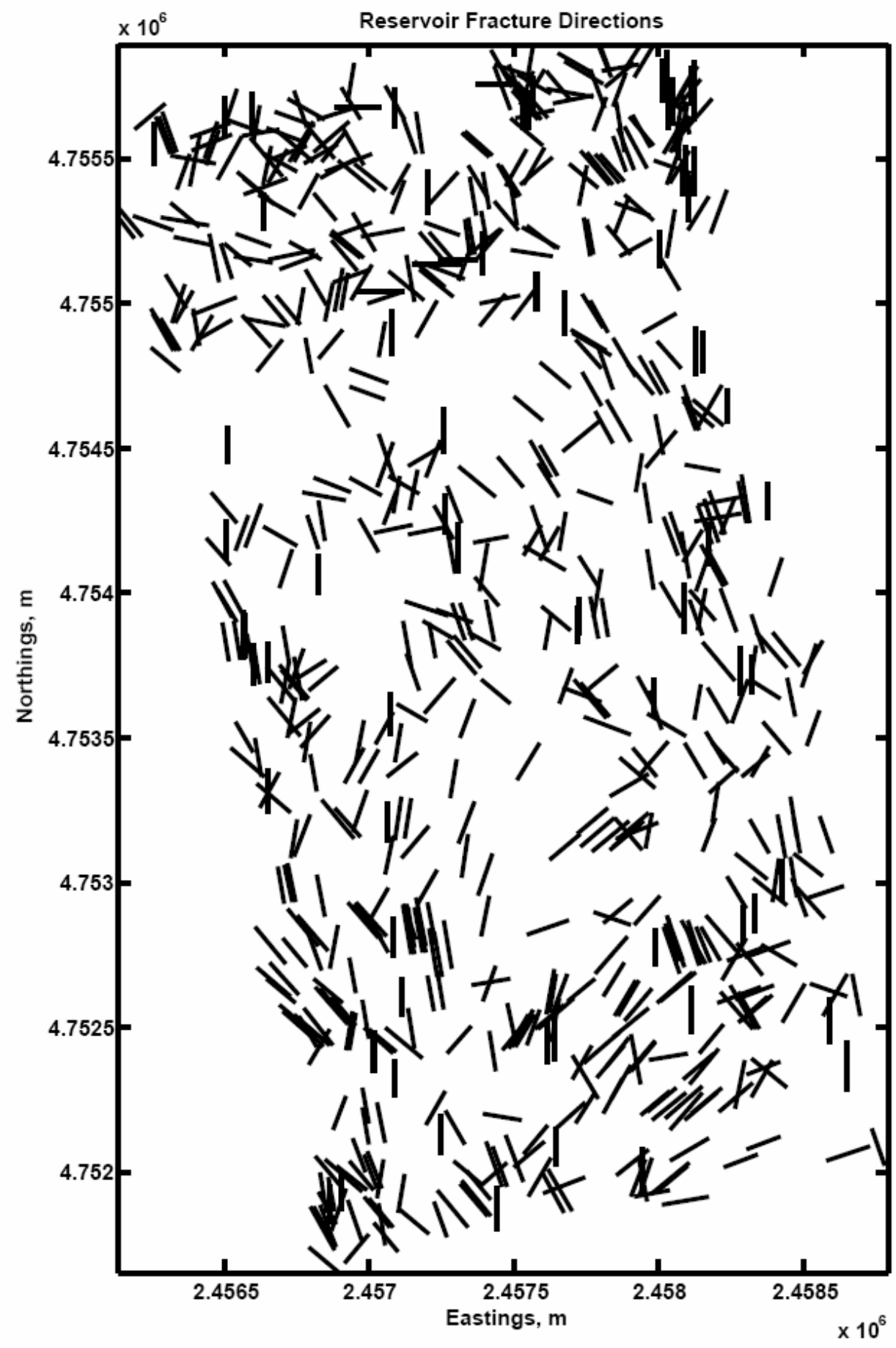

Figure 8. 
Faults Derived from Seismic and Scattering Index Fracture Orientation
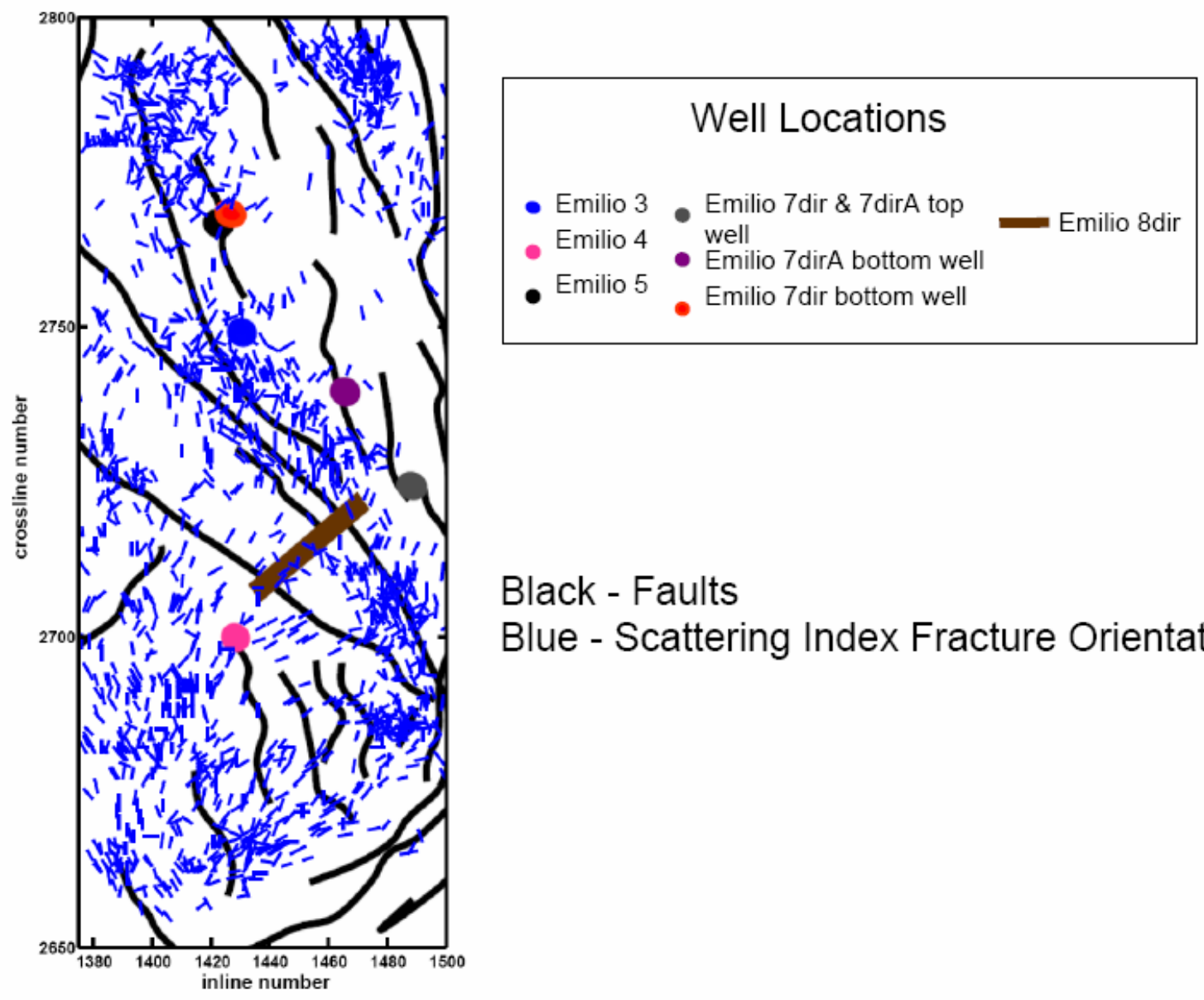

Black - Faults

Blue - Scattering Index Fracture Orientation

Figure 9. 

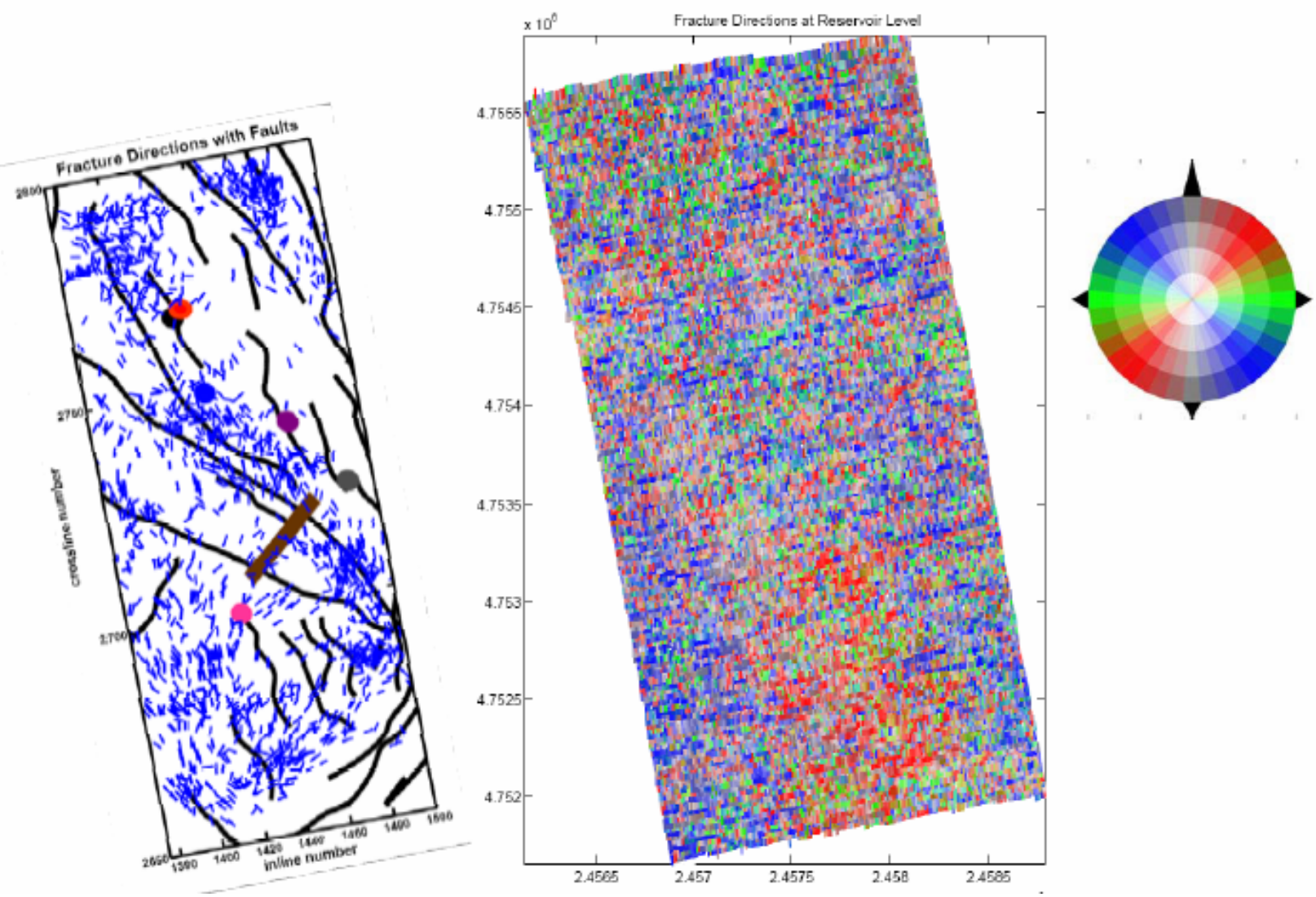

Figure 10. 


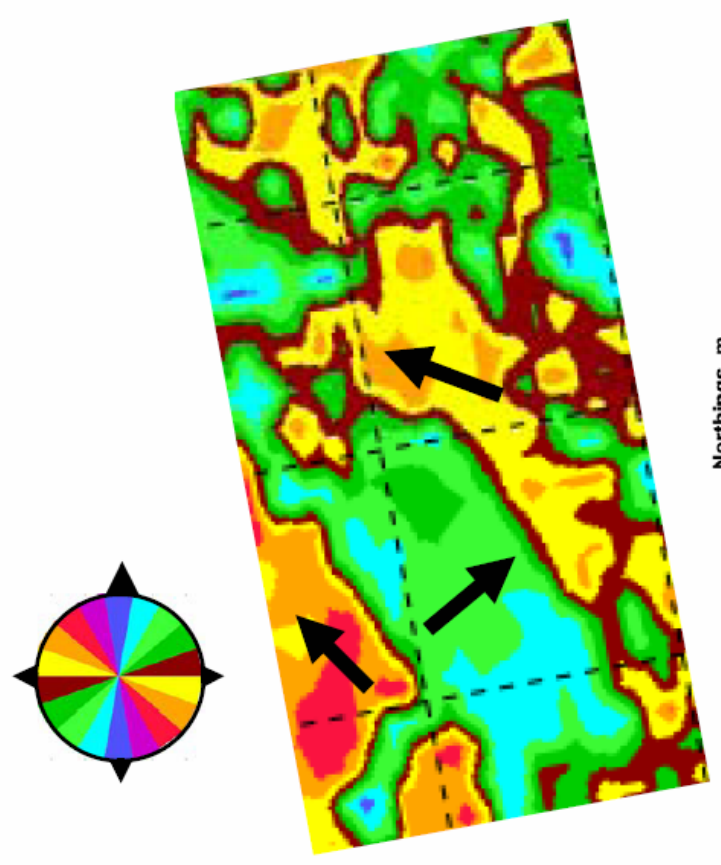

PS Fast Azimuth Fracture Orientation

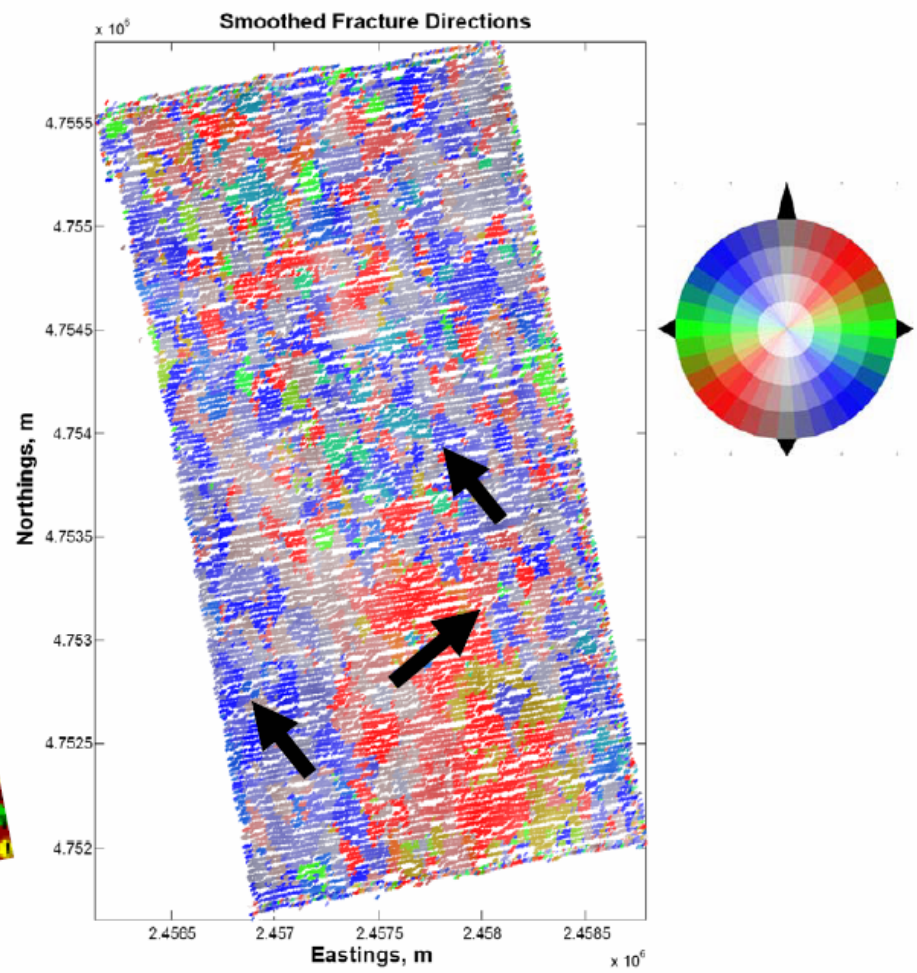

PP Scattering Index Fracture Orientation

Figure 11. 


\section{Well derived fracturing directions for 3 wells}

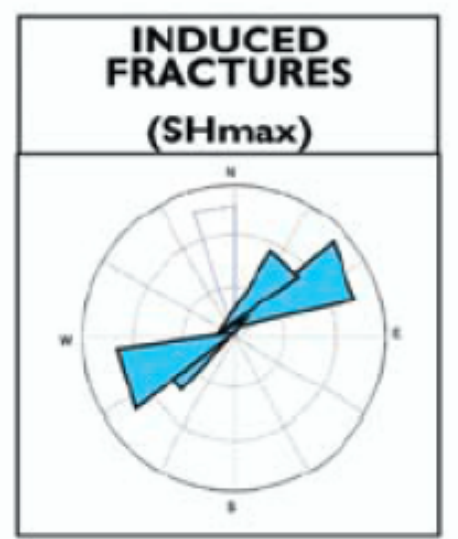

Emilio 7dir

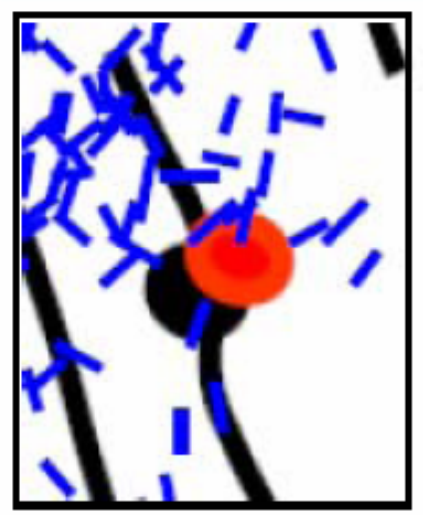

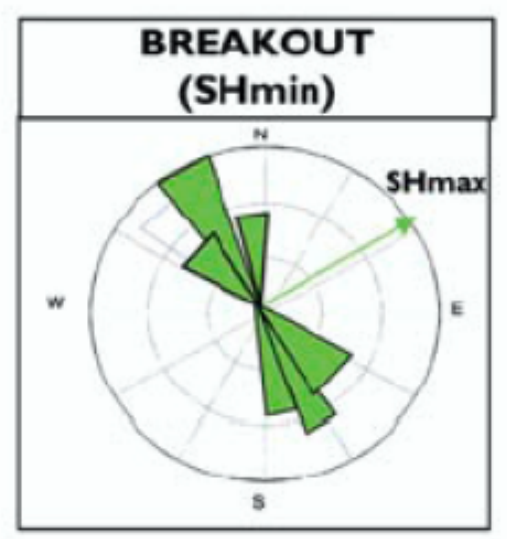

Emilio 4

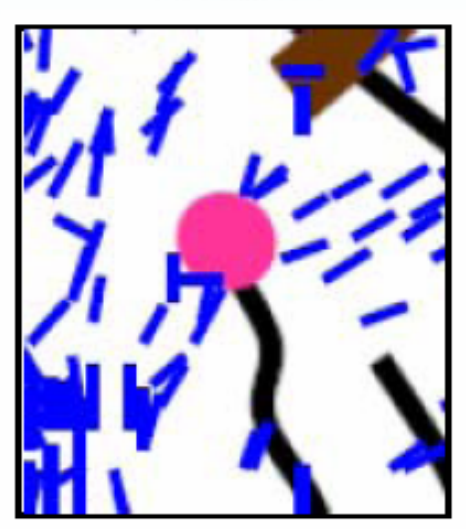

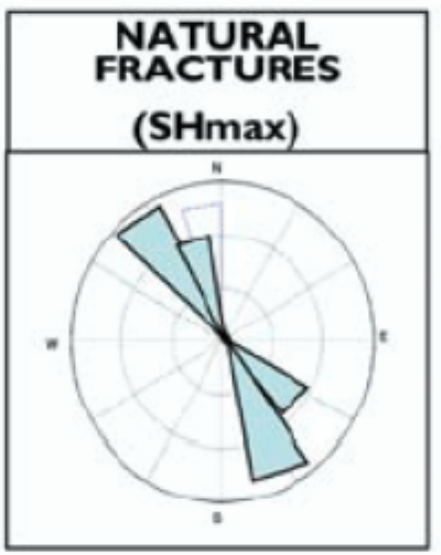

Emilio 8dir

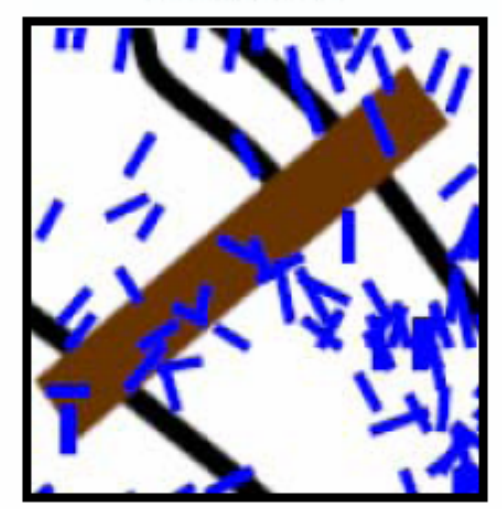

Close-up of scattering index directions at each well

Figure 12. 